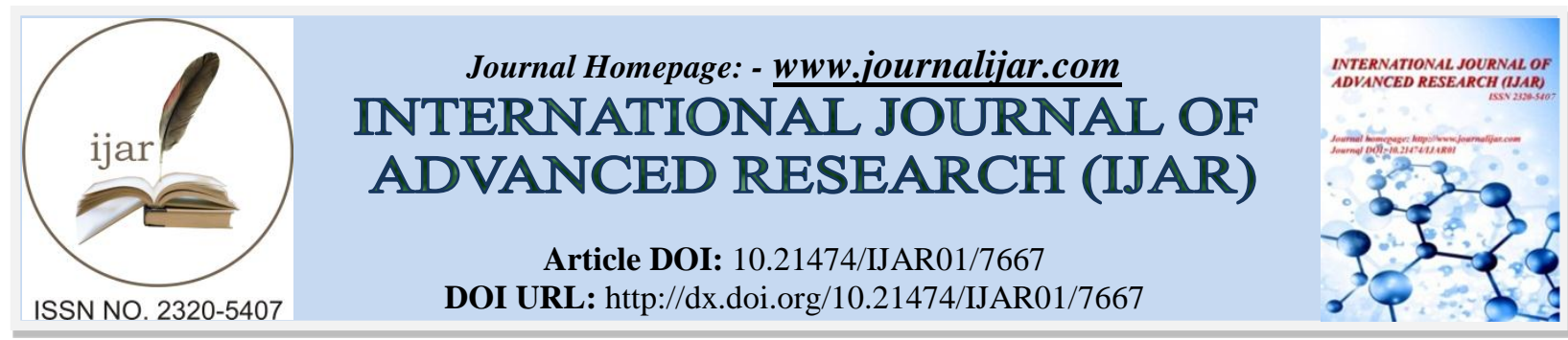

RESEARCH ARTICLE

\title{
SIMULINK APPROACH FOR RESTORATION OF RATED FREQUENCY IN INTERCONNECTED POWER SYSTEM AFFECTED BY LOADING USING ANN.
}

1. Phd scholar, JNU Jodhpur.

\author{
Rashmi Kalla $^{1}$ and G. K. joshi ${ }^{2}$.
}

2. Principal, Vyas Institute of Engineering and Technology; Former Professor and HOD Electrical Engineering, MBM Engg. College.

\section{Manuscript Info}

Manuscript History

Received: 05 July 2018

Final Accepted: 07 August 2018

Published: September 2018

Keywords:-

Overloading, Underloading, Restoration of normal frequency, Simulink, ANN.

\section{Abstract}

The aim of the present work is to obtain restoration of rated frequency in an interconnected power System which has undergone changes due to overloading as well as under loading. For the purpose a Simulink approach has been used wherein the frequency becomes less than rated frequency i.e. $50 \mathrm{~Hz}$ during overload conditions, while in the case of under loading it shows values of frequency greater than rated frequency level. The disturbed frequency is restored to normal frequency by reducing the feedback path gain while during under loading feedback path gain is increased to obtain the return of normal rated frequency. In each case the ANN is developed and later trained. After training the ANN provides the necessary feedback path gains for unknown levels of overloadings and under loadings respectively. Eventually the outcomes suggested by ANN were tested on the Simulink for which the Simulink shows the restoration of frequency. Copy Right, IJAR, 2018,. All rights reserved.

\section{Introduction:-}

The frequency of the alternator suffers adverse consequences both during overloading and under loading of conditions. Overloading causes reduction of frequency below its rated value $50 \mathrm{~Hz}$ while under loading causes increase in frequency beyond $50 \mathrm{~Hz}$.Under both the conditions it is necessary to bring back the disturbed frequency to its normal frequency i.e. $50 \mathrm{~Hz}$. The present paper uses Simulink approach on MATLAB to establish recovery of frequency to its normal value. Once, it is disturbed either due to overloading or under loading.

Authors Ping Si Aiguo Patrick Hu Simon Malpas Ping Si, Aiguo Patrick Hu, Simon Malpas and David Budgett in their work on " A Frequency control Method for Regulating Wireless Power to Implantable Devices " [1] have suggested the control of flow of power to implantable medical devices via wireless technique just by changing the operating frequency. The operating frequency is changed by means of altering the effective value of tuning capacitance. Also, the alteration in tuning capacitance is achieved through soft switched phase control. The paper therefore, asserts the co - relation of frequency and power. Authors M. Okamura, Y. O-ura, S. Hayashi , K. Uemura and F. Ishiguro in their work on "A new power flow model and solution method including load and generator characteristics and effects of system control devices" [2] have given an advanced Newton's method to achieve exact and working solutions for new steady state that would be available just after the disturbance. They have been successful in combining the steady state model with dynamic simulation program. The authors make use of load, generator characteristics and influence of control devices in obtaining the solution. Authors İlhanKocaarslan 
and ErtuğrulÇam in their work "Fuzzy logic controller in interconnected electrical power systems for loadfrequency control" [4] have provided a fuzzy gain scheduled proportional and integral (FGPI) controller for load frequency control of two area interconnected power system. It is shown through simulations of power system that FPGI controller is good enough for damping out oscillations which results from load disturbances. On comparison of this controller with other controllers it was observed that proposed FPGI controller is better than existing controllers. Authors Y. Wang, R. Zhou and C. Wen in their work on " Robust load - frequency controller design for power systems" [7] have given a robust controller using Riccati approach for load frequency control in power systems. The design of robust load frequency controller needs only the bounce of the system parameters. Also, the proposed controller is asymptotically stable for all possible uncertainties. It is observed that the robust load flow controller maintains good performance despite the presence of constraints such as generation rate constant. Authors T.H.Mohamed, H.Bevrani, A.A.Hassan and T.Hiyama in their work on "Decentralized model predictive based load frequency control in an interconnected power system" [10] have shown that a proposed load frequency control performs effectively in multi area power system and it s based on model predictive control (MPC) technique. This technique is so designed that the effect of uncertainty due to governor and turbine disturbance is reduced. The result show that MPC technique proves to be robust in dealing with uncertainties of governors and load turbines in overall closed loop system. Authors IonHsiao and DongChiang in their research "Towards a theory of voltage collapse in electric power systems" [11] have pointed out that the power system blackouts are due to progressive decline of voltage at system buses. These events are known as voltage collapses. Though the mechanisms of voltage collapses are not very much clear yet this paper attempts to explain loss of stability when an equilibrium point is lost while the bifurcation of saddle node occurs. The authors have presented a simple approach of system dynamics following the bifurcation. The authors have used the results to propose a model for voltage collapses in power systems. The authors have illustrated the model by constructing a simple power system model and simulating a voltage collapse in it. Authors J.G. Nielsen, M. Newman, H. Nielsen and F. Blaabjerg in their investigation on "Control and testing of a dynamic voltage restorer (DVR) at medium voltage level"[12] have implemented a dynamic voltage restorer (DVR) on low voltage level and medium voltage level, and they have created conditions where high power sensitive loads can be protected against voltage sags. The paper provides practical test results on $10 \mathrm{KV}$ medium voltage level using DVR at Kyndby, Denmark. The DVR was designed to protect 400 KVA load from 0.5 p.u. maximum voltage sag. The effect of DVR on the system was experimentally checked over faulted and non-faulted system states for linear as well as non linear loads. Voltage sags of different durations were created using controllable low voltage (LV) breaker fed by a 630KVA distribution transformer placed upstream of the sensitive load. The authors claim that the DVR works successfully well in all operating conditions.

The paper is organised such that Section 2 discuses the development of Simulink for Automatic Load Frequency Control followed by its testing. Section 3 explains Separate Simulinks based on the concept that the overloading is incorporated by increasing the number of poles using Second order non linear expression whereas the under loading is incorporated by reducing the number of poles using linear expression. Each time the Simulink is tested by hit and trial method using certain values to address overloading and restoration of frequency afterwards. In the second case the Simulink is tested again by hit and trial method using certain values to address under loading and restoration of frequency afterwards. During test the Simulink for increasing overload needs decreasing feedback path gain to recover the disturbed frequency. Similarly the Simulink for under loading needs increasing feedback path gain to recover the disturbed frequency. Next, Section 4 describes the development of ANNs for overloading and under loading streams. Section 5 explains how the feedback data provided by ANNs for unknown levels of loadings are tested on Simulink which in turn yield restoration of normal frequency following to disturbance. It was true for both types of Simulinks. Section 6 summarises the work followed by references.

\section{Simulink for Automatic Load Frequency Control and Its Testing for Overloading:-}

A Simulink is developed on MATLAB for automatic load frequency control. This is because the frequency is required to be maintained at rated value as and when it becomes high or low due to changes in load demand. The Simulink is tested for increasing values of load multiplier and it is observed that the frequency is decreased with increasing load demand. The disturbed state of frequency due to overloading was restored back to normal frequency that is $50 \mathrm{~Hz}$ by decreasing feedback multiplier. The test results have been observed and given in next section. The ANN is developed on MATLAB. On the basis of test results from the Simulink the ANN is trained. The ANN started giving results for unknown values of load multiplier as well as feedback multiplier.

The Simulink model and its test results are as follows: 


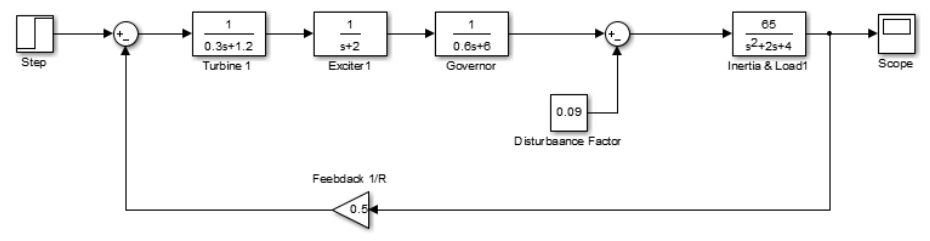

Fig. 1:-Simulink model for Automatic Load Frequency Control for overloading

The testing data of Simulink are given in table 1 for desired frequency $50 \mathrm{~Hz}$

Table 1:-Test results from Simulink

\begin{tabular}{|c|c|c|c|}
\hline S.No. & $\begin{array}{c}\text { Load Multiplier } \\
\text { (Increasing) }\end{array}$ & $\begin{array}{c}\text { Feedback Multiplier } \\
\text { (Decreasing) }\end{array}$ & Output \\
\hline 1 & $1 /\left(\mathrm{s}^{2}+2 \mathrm{~s}+4.0\right)$ & 0.09 & $50 \mathrm{~Hz}$ \\
\hline 2 & $1 /\left(\mathrm{s}^{2}+2 \mathrm{~s}+4.1\right)$ & 0.09 & $49 \mathrm{~Hz}$ \\
\hline 3 & $1 /\left(\mathrm{s}^{2}+2 \mathrm{~s}+4.1\right)$ & 0.08 & $50 \mathrm{~Hz}$ \\
\hline 4 & $1 /\left(\mathrm{s}^{2}+2 \mathrm{~s}+4.2\right)$ & 0.08 & $49 \mathrm{~Hz}$ \\
\hline 5 & $1 /\left(\mathrm{s}^{2}+2 \mathrm{~s}+4.2\right)$ & 0.04 & $50 \mathrm{~Hz}$ \\
\hline 6 & $1 /\left(\mathrm{s}^{2}+2 \mathrm{~s}+4.3\right)$ & 0.04 & $49 \mathrm{~Hz}$ \\
\hline 7 & $\left.1 / \mathrm{s}^{2}+2 \mathrm{~s}+4.3\right)$ & 0.025 & $50 \mathrm{~Hz}$ \\
\hline 8 & $1 /\left(\mathrm{s}^{2}+2 \mathrm{~s}+4.4\right)$ & 0.025 & $48 \mathrm{~Hz}$ \\
\hline 9 & $1 /\left(\mathrm{s}^{2}+2 \mathrm{~s}+4.4\right)$ & 0.005 & $50 \mathrm{~Hz}$ \\
\hline 10 & $1 /\left(\mathrm{s}^{2}+2 \mathrm{~s}+4.5\right)$ & 0.005 & $48 \mathrm{~Hz}$ \\
\hline 11 & $1 /\left(\mathrm{s}^{2}+2 \mathrm{~s}+4.5\right)$ & 0.0012 & $50 \mathrm{~Hz}$ \\
\hline 12 & $1 /\left(\mathrm{s}^{2}+2 \mathrm{~s}+4.6\right)$ & 0.0012 & $48 \mathrm{~Hz}$ \\
\hline 13 & $1 /\left(\mathrm{s}^{2}+2 \mathrm{~s}+4.6\right)$ & 0.0008 & $50 \mathrm{~Hz}$ \\
\hline
\end{tabular}

The monitor outcome of some of above test results are indicated in following figures. The graphs show the disturbed result due to increase in load and restoration of same due to decrease in feedback multiplier.

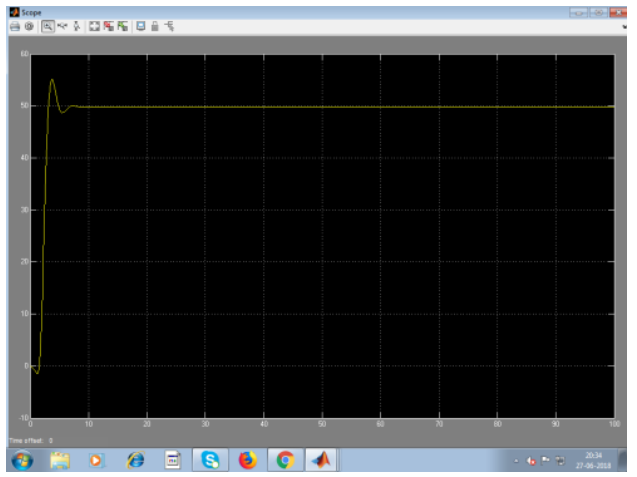

Fig. 2:-Normal Frequency for load multiplier 4.0 and feedback multiplier 0.09 


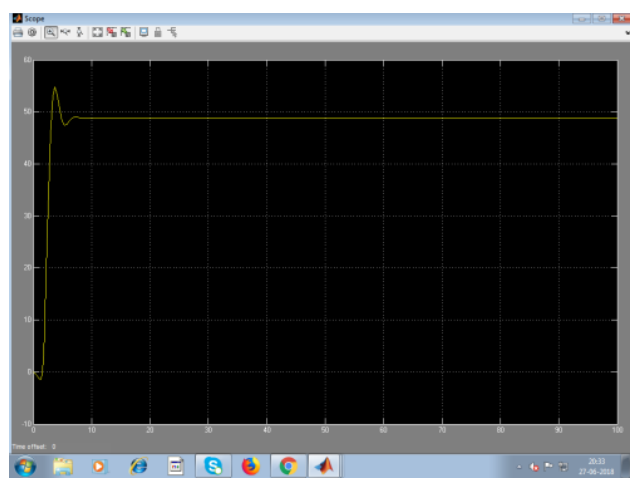

Fig.3:-Disturbed frequency for load multiplier 4.1 and feedback multiplier 0.09

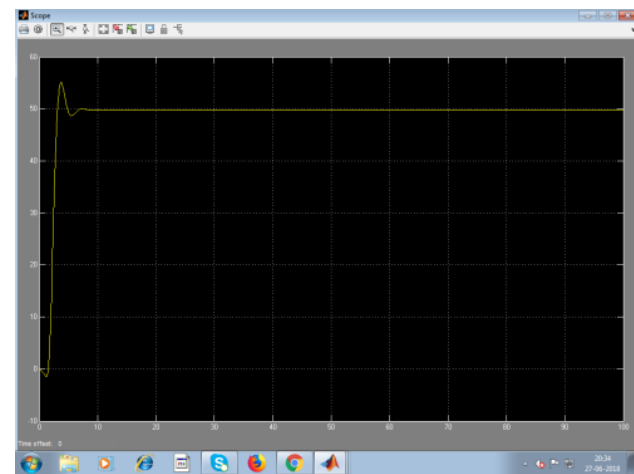

Fig. 4:-Restored Frequency for load multiplier 4.1 and feedback multiplier 0.08

It is observed that the feedback multiplier is decreased to get restoration of normal frequency which is earlier disturbed due to increase in load multiplier.

Simulink for Automatic Load Frequency Control and its testing during under loading:-

The Simulink is tested for decreasing values of load multiplier and it is observed that the frequency is increased with decreasing load demand. The disturbed state of frequency due to under loading was restored back to normal frequency that is $50 \mathrm{~Hz}$ by increasing feedback multiplier.

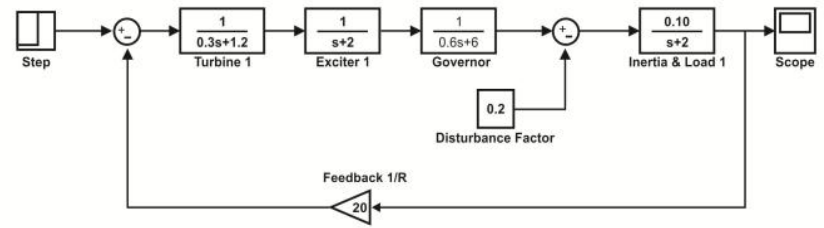

Fig. 5:-Simulink model for testing Automatic Load Frequency Control for under loading

The testing data of Simulink are given in table 2 for desired frequency $50 \mathrm{~Hz}$

Table 2:-Test results from Simulink

\begin{tabular}{|c|c|c|c|}
\hline S. No. & $\begin{array}{c}\text { Load Multiplier } \\
\text { (Decreasing) }\end{array}$ & $\begin{array}{c}\text { Feedback Multiplier } \\
\text { (Increasing) }\end{array}$ & Output \\
\hline 1 & $1 /(\mathrm{S}+2)$ & 0.71 & $50 \mathrm{~Hz}$ \\
\hline 2 & $1 /(\mathrm{S}+1.85)$ & 0.71 & $54 \mathrm{~Hz}$ \\
\hline 3 & $1 /(\mathrm{S}+1.85)$ & 0.725 & $50 \mathrm{~Hz}$ \\
\hline 4 & $1 /(\mathrm{S}+1.70)$ & 0.725 & $60 \mathrm{~Hz}$ \\
\hline 5 & $1 /(\mathrm{S}+1.70)$ & 0.74 & $50 \mathrm{~Hz}$ \\
\hline 6 & $1 /(\mathrm{S}+1.55)$ & 0.74 & $54 \mathrm{~Hz}$ \\
\hline 7 & $1 /(\mathrm{S}+1.55)$ & 0.77 & $50 \mathrm{~Hz}$ \\
\hline
\end{tabular}




\begin{tabular}{|c|l|l|c|}
\hline 8 & $1 /(\mathrm{S}+1.40)$ & 0.77 & $57 \mathrm{~Hz}$ \\
\hline 9 & $1 /(\mathrm{S}+1.40)$ & 0.79 & $50 \mathrm{~Hz}$ \\
\hline 10 & $1 /(\mathrm{S}+1.25)$ & 0.79 & $54 \mathrm{~Hz}$ \\
\hline 11 & $1 /(\mathrm{S}+1.25)$ & 0.82 & $50 \mathrm{~Hz}$ \\
\hline 12 & $1 /(\mathrm{S}+1.10)$ & 0.82 & $57 \mathrm{~Hz}$ \\
\hline 13 & $1 /(\mathrm{S}+1.10)$ & 0.86 & $50 \mathrm{~Hz}$ \\
\hline
\end{tabular}

The monitor outcome of some of above test results are indicated in following figures. The graphs show the disturbed result due to change in load and restoration of same due to increase in feedback multiplier.

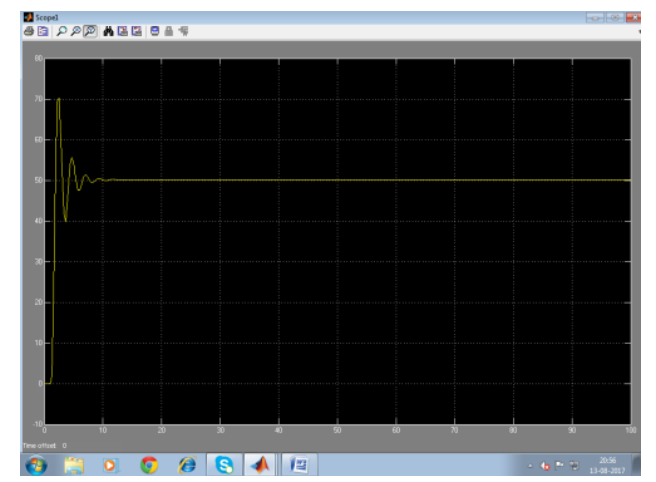

Fig.6:-Normal Frequency for load multiplier $1 /(s+2)$ and feedback multiplier 0.71

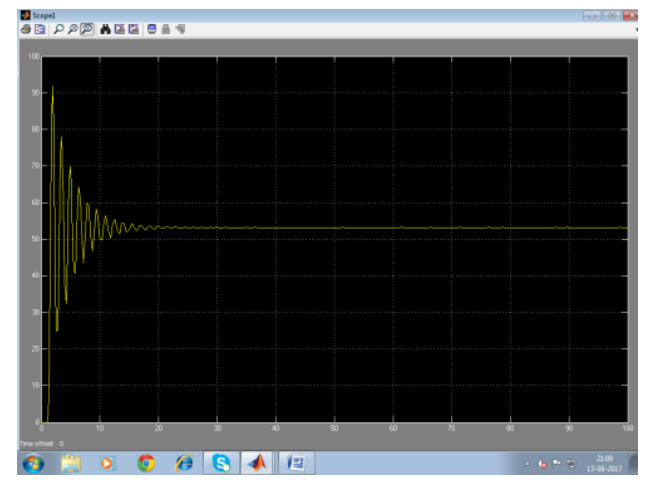

Fig.7:-Disturbed frequency for load multiplier $1 /(\mathrm{s}+1.85)$ and feedback multiplier 0.71

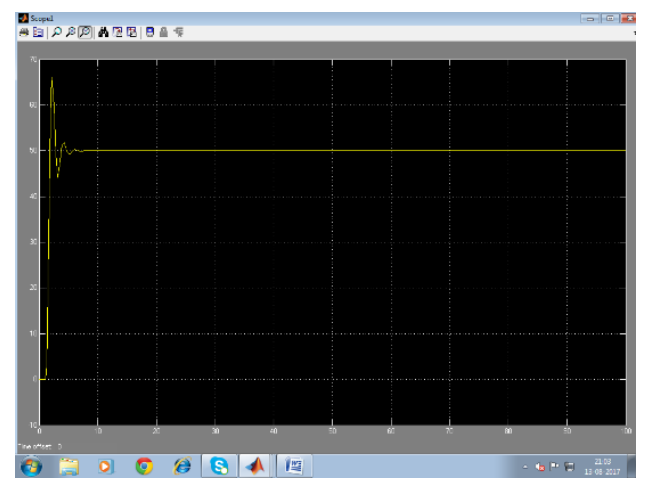

Fig. 8:-Restored Frequency for load multiplier $1 /(\mathrm{s}+1.85)$ and feedback multiplier 0.725

It is interesting to note that increasing load leads to decrease in Frequency, while decrease in load leads to increase in Frequency. 
By using ANN it could be possible to avoid hit and trial testing on Simulink and it straight away provided the required feedback in either conditions.

\section{Development of ANN for frequency disturbance due to over loading and under loading:-}

An ANN has been developed for knowing the outcome of Simulink without testing it , but by testing ANN only, for the purpose the ANN is trained by test results of Simulink data. The ANN program is as under. It is followed by its testing. The ANN program for Automatic Load Frequency Controller is as under $\mathrm{a}=0.85: 0.05: 1.15$;

$\mathrm{b}=[0.16 ; 0.2 ; 0.24 ; 0.27 ; 0.30 ; 0.35 ; 0.4]$;

(n................

net=newff(p,t,5);

net=train(net,p,t);

$\mathrm{y}=\operatorname{sim}($ net, $\mathrm{a})$;

gensim(net)

The ANN training:-

The ANN is trained by test results of Simulink data.

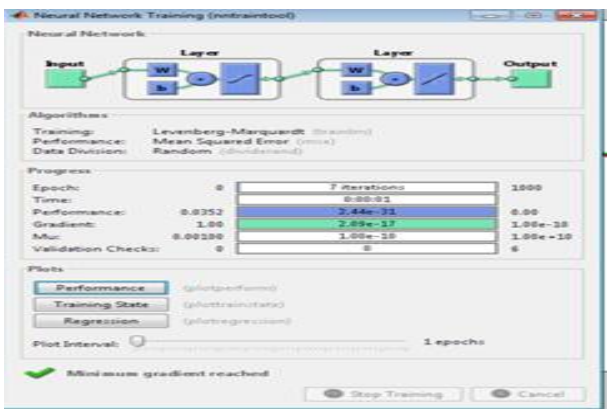

Fig.9:-ANN for implementing Automatic Load Frequency Control

\section{The ANN Performance:-}

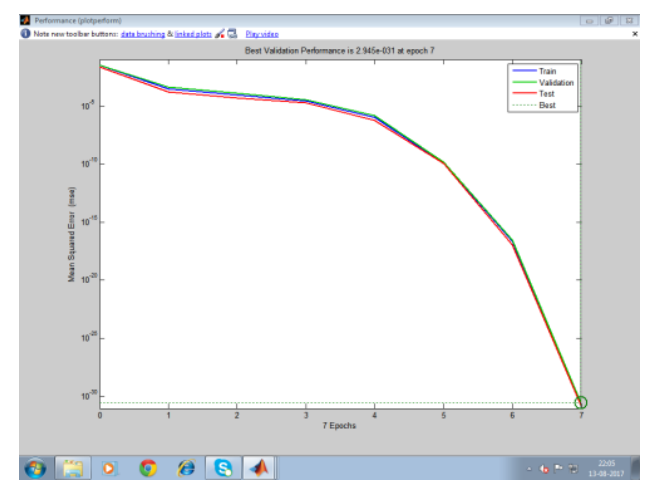

Fig.10:-Performance of ANN for implementing Automatic Load Frequency Control 


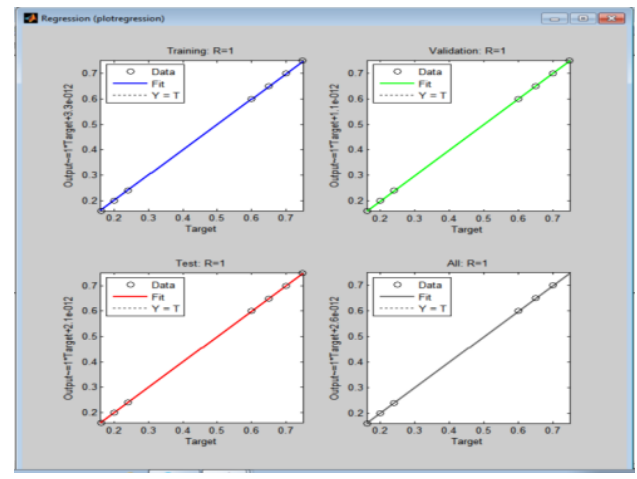

Fig.11:-Regression plots of trained ANN for Automatic Load Frequency Control

Outcomes of ANN for some unknown levels of overloading:-

Having trained the ANN for increasing load demand and accordingly reducing frequency. The ANN was tested for unknown levels of overloading. The overloading lead to reduction in frequency. It was than restored by increasing feedback multiplier. The results are shown as under.

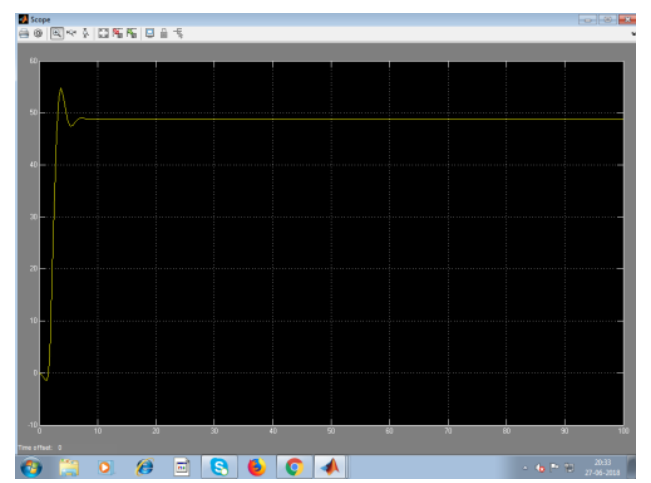

Fig. 12:-Test Result for Disturbed frequency at load multiplier 4.15 and feedback multiplier 0.09

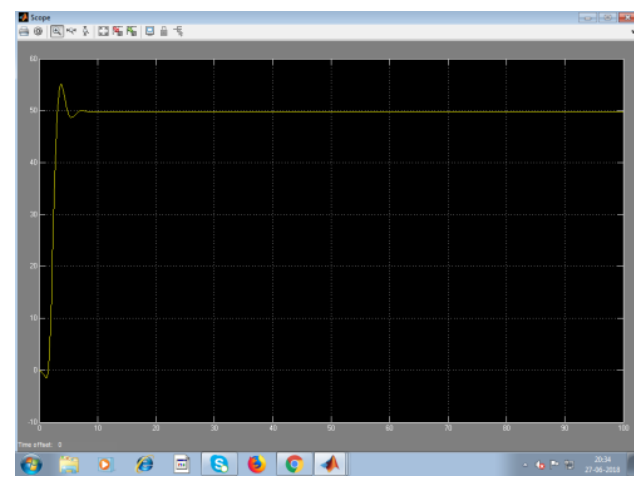

Fig. 13:-Test Result for Restored frequency at load multiplier 4.15 and feedback multiplier 0.085

\section{Conclusion:-}

Independent Simulink models one for increasing load demand and other for decreasing load demand were developed on MATLAB platform. The Simulink for increasing load demand exhibited lowering of frequency from its rated frequency level i.e. $50 \mathrm{~Hz}$ due to increase in load multiplier. But its restoration to normal value could be achieved by decreasing the feedback multiplier. Similarly, the Simulink for decreasing load demand exhibited increasing of frequency from its rated level. This was due to decrease in load multiplier. But its restoration was achieved by increasing the feedback multiplier. The values so received by trial and error approach were used as the basis for training the artificial neural network (ANN). Later when ANN got trained it was tested for the unknown 
vales of load multiplier and feedback multiplier. It was found that these multiplier help achieve restoration of normal frequency which is disturbed due to overloading or under loading conditions

\section{References:-}

1. Ping Si Aiguo Patrick Hu Simon Malpas Ping Si, Aiguo Patrick Hu, Simon Malpas and David Budgett, "A Frequency control Method for Regulating Wireless Power to Implantable Devices ", IEEE Transactions on Biomedical Circuits and Systems, Vol. 2, Issue: 1, March 2008, pp $22-29$

2. M. Okamura, Y. O-ura, S. Hayashi, K. Uemura and F. Ishiguro, " A new power flow model and solution method including load and generator characteristics and effects of system control devices", IEEE Transactions on Power Apparatus and Systems , Vol. 94, Issue: 3, May 1975, pp 1042 - 1050 , Print ISSN: 0018-9510

3. P.M. Anderson and M. Mirheydar, "An adaptive method for setting under frequency load shedding relays", IEEE Transactions on Power Systems, Vol. 7, Issue: 2, May 1992 , pp $647-655$

4. İlhanKocaarslan and ErtuğrulÇam, "Fuzzy logic controller in interconnected electrical power systems for loadfrequency control", ELSEVIER, International Journal of Electrical Power \& Energy Systems, Vol. 27, Issue 8, October 2005, pp 542-549

5. P. Kessel and H. Glavitsch, "Estimating the Voltage Stability of a Power System", IEEE Transactions on Power Delivery, Vol. 1, Issue: 3, July 1986 ,pp 346 - 354

6. C. Concordia, L.H. Fink and G. Poullikkas, "Load shedding on an isolated system", IEEE Transactions on Power Systems ,Vol. 10, Issue: 3, Aug. 1995, pp 1467 - 1472

7. Y. Wang, R. Zhou and C. Wen, " Robust load - frequency controller design for power systems", IET digital library, Vol. 140, Issue 1, January 1993, p. 11 - 16, Print ISSN 0143-7046, Online ISSN 2053-7921

8. A .von Jouanne_and B. Banerjee, "Assessment of voltage unbalance", IEEE Transactions on Power Delivery Vol. 16, Issue: 4, Oct 2001, pp 782 - 790 , Print ISSN: 0885-8977

9. P.-A. Lof, G. Andersson and D.J. Hill, "Voltage stability indices for stressed power systems", : IEEE Transactions on Power Systems ,Vol. 8, Issue: 1, Feb. 1993 , pp 326 - 335

10. T.H.Mohamed, H.Bevrani, A.A.Hassan and T.Hiyama, "Decentralized model predictive based load frequency control in an interconnected power system", ELSEVIER, Energy Conversion and Management, Vol. 52, Issue 2, February 2011, Pp 1208-1214

11. IanDobsonHsiao and DongChiang, "Towards a theory of voltage collapse in electric power systems", ELSEVIER, Systems \& Control Letters, Vol. 13, Issue 3, September 1989, Pp 253-262

12. J.G. Nielsen, M. Newman, H. Nielsen and F. Blaabjerg, "Control and testing of a dynamic voltage restorer (DVR) at medium voltage level", IEEE Transactions on Power Electronics, Vol. 19, Issue: 3, May 2004 , pp $806-813$. 